\title{
Modelo de gestión empresarial en la dinamización de las MYPEs de confecciones de un parque industrial
}

\section{Business management model in the revitalization of MYPEs of clothing of an Industrial Park}

\author{
Jesús Fernando Bejarano Auqui ${ }^{1 \mathrm{ab}}$ \\ Universidad Peruana Unión, Lima, Perú ${ }^{1}$
}

Recibido: 13 de noviembre de 2020

Aceptado: 12 de mayo de 2021

\begin{abstract}
Resumen
La administración de la actividad económica de los propietarios de las MYPEs está basada en la experiencia empresarial a expensas de los conocimientos académicos, que los limitan desarrollar una Gestión Empresarial (GE) con mayor competitividad. Una solución a este problema es el empleo del modelo de GE que dinamizara las actividades productivas de estas organizaciones. El objetivo de la investigación fue diseñar un modelo de gestión empresarial basado en las teorías críticas del pensamiento administrativo para las MYPEs de confecciones del Parque Industrial Ate-Vitarte. El tipo de estudio fue descriptivo cuantitativo. La muestra estuvo representada por 101 empresarios. Los resultados del análisis de regresión lineal múltiple evidenciaron que el $\mathrm{r}^{2}$ ajustado de $\mathrm{Gci}=0.898, \mathrm{Gv}=0.781$. Ge $=0.679, \mathrm{Gf}=0.737 \mathrm{y} \mathrm{Td}=0.495$. Conclusión el modelo GE motivara a los empresarios ser agentes de cambio en su entorno social y económico, usar los elementos de la administración y criterios del pensamiento administrativo con mayor conocimiento e información. Los hallazgos de sus conceptos, teorías y utilidad metodológica servirán para la construcción de
\end{abstract}

Palabras clave: Gestión empresarial, gestión del conocimiento e información, gestión del valor, gestión económica, gestión financiera, toma de decisiones y dinamización de la actividad económica de las MYPEs

\begin{abstract}
The administration of the economic activity of the owners of the MYPEs is based on business experience at the expense of academic knowledge, which limits them to develop a Business Management (BM) with greater competitiveness. A solution to this problem is the use of the BM model that will boost the productive activities of these organizations. Therefore, the objective of the research was to design a business management model based on critical theories of administrative thought for the MYPEs of clothing in the Ate-Vitarte Industrial Park. The type of study was descriptive quantitative. The sample was represented

${ }^{\mathrm{a}}$ Correspondencia al autor

E-mail: jesusbejarano@upeu.edu.pe

${ }^{b}$ La presente investigación se basa en la tesis de maestría del autor: http://hdl.handle.net/20.500.12840/3338
\end{abstract}


by 101 businessmen. The results of the multiple linear regression analysis showed that the adjusted $\mathrm{r} 2$ of $\mathrm{Gci}=0.898, \mathrm{Gv}=0.781 . \mathrm{Ge}=0.679, \mathrm{Gf}=0.737$ and $\mathrm{Td}=0.495$. Conclusion the GE model will motivate entrepreneurs to be agents of change in their social and economic environment, using the elements of administration and criteria of administrative thought with greater knowledge and information. The findings of their concepts, theories and methodological utility will serve for the construction of future lines of research that will cover other empty spaces still to be investigated.

Keywords: Business management, knowledge and information management, value management, economic management, financial management, decision making and revitalization of the economic activity of the MYPEs

\section{Introducción}

A través del tiempo los modelos de las teorías del pensamiento administrativo han venido siendo usados por los diversos sectores económicos para revertir sus paradigmas de administrar sus empresas a pesar de sus postulados heterogéneos. Hernández (2011) considera que las teorías científicas de la administración a pesar de presentar dificultades de integrar sus teorías en la gestión empresarial debido a su heterogeneidad de sus pensamientos e información siguen siendo importantes en la integración de los recursos materiales, técnicos y humanos.

En el entorno del siglo XXI, las organizaciones se ven en la necesidad de cambio constante y rápido, debido a que el mercado globalizador es cada vez más competitivo y requiere de empresarios con visión en gestión empresarial, que aprendan de sus errores y se planteen nuevos modelos de administración, mayor dinamismo en la productividad con mayor valor agregado, liderazgo e innovación (Morais-Storz, Stoud \& Berild, 2018). Ante la necesidad de gestionar el cambio y promover la mejora continua, las teorías de la administración, si bien es cierto que han estado solucionando los problemas empresariales, sin embargo, estos modelos no han sido lo suficientemente exitosos para muchas empresas que tras períodos de grandes logros han caído en fases de problemas coyunturales como lo que actualmente están pasando las economías del mundo ante la pandemia mundial de la COVID-19, cuyo impacto en la dinamización de la actividad económica de las organizaciones se hizo distante e incierta en las inversiones, producción, ingreso y empleo.

En este escenario, el articulo cubrirá el espacio vacío que en la literatura existente de los modelos del pensamiento administrativo y seguidores de estas corrientes epistemológicas se estudian muy poco en: Gestión del conocimiento me información, gestión del valor, 
gestión financiera, gestión económica y toma de decisiones. La importancia del artículo radica que estas características al ser considerados por los propietarios de las MYPEs, impactaran en la gestión empresarial con mayor productividad y calidad humana. El objetivo del artículo es diseñar un modelo de gestión empresarial basado en las teorías críticas del pensamiento administrativo para las MYPEs de confecciones del Parque Industrial AteVitarte.

\section{Modelos del pensamiento administrativo}

Sin duda que la escuela de la Administración Científica, tuvo como su principal exponente a Frederick Taylor, quien enfatizó el aumento de la productividad mediante una mayor eficiencia en la producción y la aplicación del método científico. Resaltó el diseño del trabajo productivo a través del estudio de tiempo-movimiento (Niebel \& Freivalds, 2009). Resalto la necesidad de racionalizar los métodos de trabajo, mediante la división del trabajo con el fin de maximizar la eficiencia de la mano de obra maquinarias y herramientas; y establecer un salario en función al tiempo de producción (Hodson, 2001). Estableció premios e incentivos para los trabajadores que cumpliesen con las normas y superen las metas de trabajo. Taylor catalogaba al hombre como un Homo Economicus, influenciado y motivado por las recompensas salariales y materiales.

La Teoría Clásica de la Administración (TCA) de Henry Fayol. identifica los principios y conocimientos que originan en la administración efectiva. Plantea que los principios de la administración son intangibles y afectan la conducta administrativa. Orienta el aumento de la eficiencia de la organización productora de bienes y servicios a través de la interrelación entre los mandos jerárquicos de la empresa. Velásquez, (2002) menciona que Henry Fayol, como creador de TCA se preocupó por definir las funciones básicas de la empresa, el concepto de administración: planear, organizar, dirigir, y controlar. Según (Espinoza, 2009) Fayol, planteo los llamados principios generales de la administración que van desde la división del trabajo hasta espíritu de equipo como procedimientos universales, para que la gerencia tenga mayores elementos de juicio para administrar el negocio. Además, Fayol dividió las operaciones administrativas en principales funciones de operaciones técnicas, comerciales, financieras, de seguridad, contabilidad y gerencia.

La teoría de la burocracia de Max Weber (1864-1920), quien considero que la burocracia refiere a características del diseño organizacional. Planteó que la burocracia es la forma más eficiente y racional que utilizaban las organizaciones con dinámicas productivas 
industriales y lograr un elevado nivel de eficiencia y control efectivo sobre el capital de trabajo, derivado de las exigencias de la sociedad moderna. El modelo burocrático enfatizo la estructura organizacional cuyas funciones son de especialización, estabilidad, racionalidad y productividad (Gómez, 2008). El modelo de las relaciones humanas de Munsterberg y Elton Mayo (1924-1932), resalta que una organización con identidad social y económica impulsa mejorar las condiciones de las relaciones humanas y sociales (Ponce \& Salas, 2014). El pensamiento administrativo del comportamiento de Abrahán Maslow y Douglas Mc Gregor (1908-1970) considera que las relaciones de trabajo deben estar orientados a los niveles de satisfacción de las necesidades de trabajo (Chiavenato, 1999).

El paradigma teórico X Y de Douglas Mc Gregor, postula que la persona X realiza lo que la empresa pide que haga. Es un mercantilista de su fuerza de trabajo. Mientras la persona $\mathrm{Y}$ es un trabajador innovador, tiene un estilo de administración abierto y democrático (Medina \& Ávila, 2002). Por su parte la teoría estructuralista de Burnham (1950), menciona que los niveles en la estructuración jerárquica de las empresas son eficientes en la medida como se estructuran sus niveles gerenciales y niveles técnicos (Beltán, 2020). La teoría de los sistemas de Von Bertalanffy (1950-1968), enfatiza la entropía como una tendencia que los sistemas se desgastan por el desorden de sus sistemas (Torres, 2014; Velásquez, 2002). Según el modelo de las contingencias de Dill, Starbuck, Thompson, Lawrence y Burns en su teoría el funcionamiento de una organización depende de la interacción con el entorno en base a la influencia tecnológica, estructura y comportamiento (Hernández \& Rodríguez, 2012). Finalmente, el modelo de la teoría del desarrollo organizacional de Bennis, Blake y Shepard (1958), enfatiza que el desarrollo planificado sobre la relación individuo-organización-ambiente debe generar cambio social. 
Tabla 1

Modelos del pensamiento administrativo

\begin{tabular}{|c|c|c|c|}
\hline Teorías & Paradigmas & $\begin{array}{l}\text { Objetivo } \\
\text { organizacional }\end{array}$ & Hipótesis \\
\hline $\begin{array}{l}\text { Administración } \\
\text { científica } \\
\text { Frederick Taylor } \\
(1,856-1,915)\end{array}$ & $\begin{array}{l}\text { Se centra en la } \\
\text { departamentalización }\end{array}$ & $\begin{array}{l}\text { Aumentar la producción } \\
\text { a través de la eficiencia } \\
\text { empresarial }\end{array}$ & $\begin{array}{l}\text { La racionalización del } \\
\text { trabajo con énfasis a las } \\
\text { tareas mejora la } \\
\text { eficiencia } \\
\text { organizacional. }\end{array}$ \\
\hline $\begin{array}{l}\text { Teoría clásica de la } \\
\text { administración } \\
\text { Henry Fayol } \\
(1,841-1,925)\end{array}$ & $\begin{array}{l}\text { Postula una } \\
\text { organización } \\
\text { estructuralista }\end{array}$ & $\begin{array}{llr}\text { Maximizar } & & \text { la } \\
\text { eficiencia en } & \text { las } \\
\text { actividades de } & \text { la } \\
\text { organización } & & \end{array}$ & $\begin{array}{l}\text { La aplicación de los } \\
\text { principios y las } \\
\text { funciones de la } \\
\text { administración con } \\
\text { eficiencia mejora la } \\
\text { actividad empresarial }\end{array}$ \\
\hline $\begin{array}{l}\text { Teoría de } \\
\text { burocracia } \\
\text { Max Weber } \\
(1,864-1920)\end{array}$ & $\begin{array}{l}\text { Estructura } \\
\text { organizacional } \\
\text { formal y cerrado }\end{array}$ & $\begin{array}{l}\text { Enfatizar en la } \\
\text { organización formal } \\
\text { el enfoque de un } \\
\text { sistema } \\
\text { organizacional } \\
\text { cerrado. }\end{array}$ & $\begin{array}{l}\text { Las normas y } \\
\text { reglamentos acertados } \\
\text { reducen los errores } \\
\text { humanos permitiendo } \\
\text { que los resultados de } \\
\text { los procesos sean más } \\
\text { visibles. }\end{array}$ \\
\hline
\end{tabular}

Teoría de las relaciones humanas Hugo Munsterberg y Elton Mayo

$(1,924-1,932)$

\section{Relaciones} humanas

interpersonales en el
trabajador
Aportes principales

Racionalización

trabajo a través de

tiempo movimiento.

Establece los principios

de la administración científica: POCE.

Énfasis en la estructura organizacional, cargos y funciones.

Estableció el proceso

administrativo: PODEC

Creo 14 principios de la administración.

Estableció funciones técnicas, comerciales, financieras, seguridad y contables para las organizaciones.

Estructura funcional cerrado e importancia en la planeación y control.

Establecimientos de tipos de sociedades y autoridades.

En las relaciones interpersonales la organización es un ente de personas. $\mathrm{La}$ delegación de autoridad, autonomía del trabajador, las recompensas sociales y la producción depende de la integración social

Teoría científica del comportamiento Abrahán Maslow y Douglas Mc

Gregor

(1,908-1,970)

Teoría X y Y

Douglas Mc

Gregor

(1,906-1,964)

Teoría

estructuralista

James Burnham

$(1,950$
Satisfacción de las necesidades según sus niveles.

(n)

Motivar las La satisfacción de las relaciones de trabajo necesidades del considerando sus empleado les hará más necesidades del satisfecho. trabajador

Innovación $\quad \mathrm{y}$ creatividad de los individuos.

Estructura organizacional basado en niveles jerárquicos.

\section{Despertar a la} persona $\mathrm{X}$ el interés de valorar su participación Motivar al capital humano $\mathrm{Y}$ para desarrollar e innovar nuevas formas de gestión empresarial.

Armonía entre los Mayor comunicación propósitos organizacionales e niveles jerárquicos
$\mathrm{El}$ individuo $\mathrm{X}$ es dependiente de las ordenes de la administración.

Trabajo es como mercancía.

$\mathrm{El}$ individuo $\mathrm{Y}$ es innovador y no necesita controlado. y participación en los individuales.
En las personas orientadas en los niveles de satisfacción de las necesidades de Maslow

La teoría X lleva a las personas hacer exactamente lo que la organización pide que haga (imposición).

La teoría Y desarrolla un estilo de administración abierto y democrático.

Niveles jerárquicos de empresa:

Nivel gerencial

Nivel técnico.
Resultados

Máxima eficiencia

en la gestión

empresarial.

Máxima eficiencia en la actividad económica de la empresa.

Eficiencia máxima.

Eficiencia óptima.

Empleado satisfecho.

Búsqueda de metas.

Eficiencia y eficacia (n) 


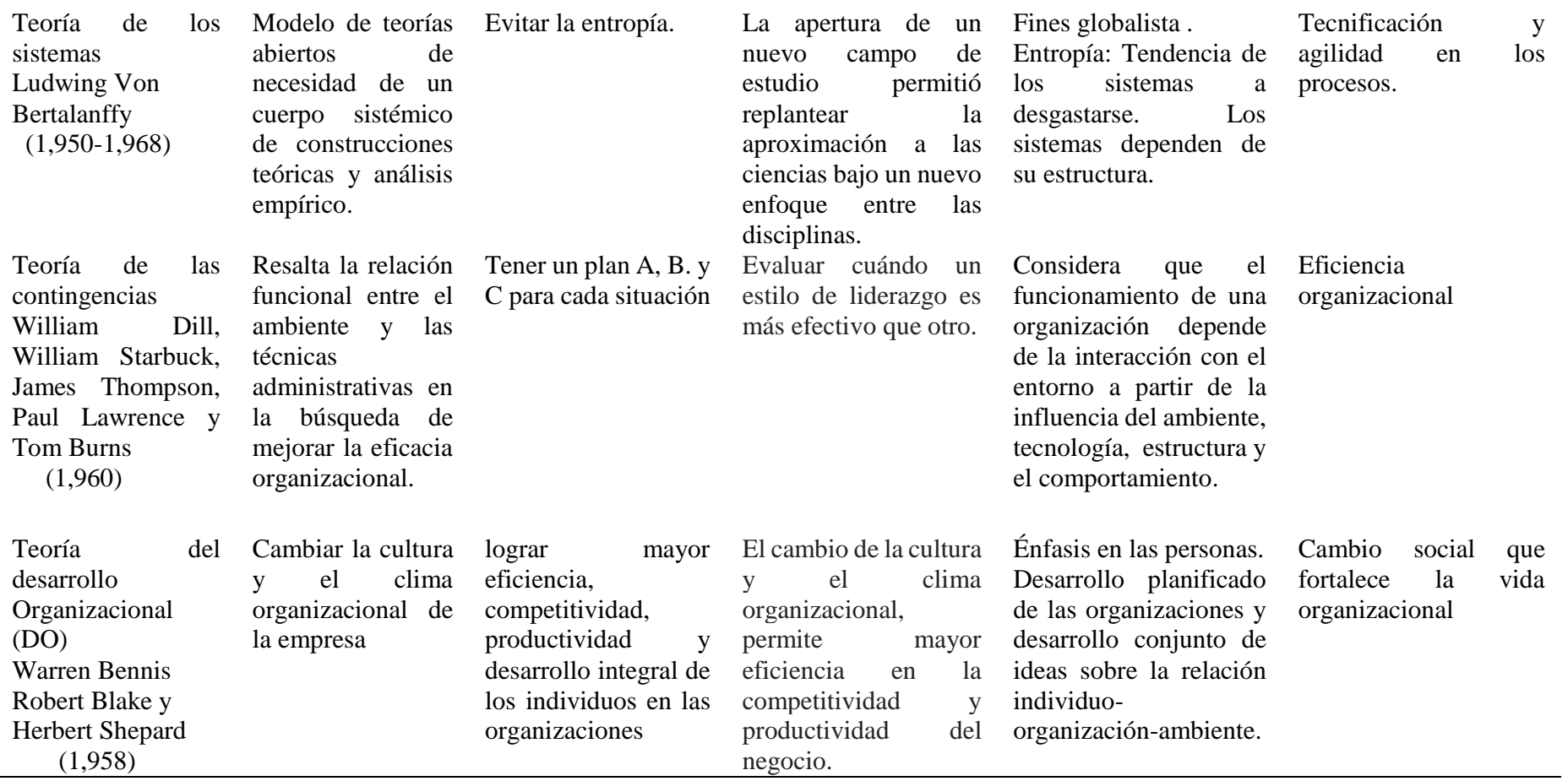

\section{Gestión empresarial}

\section{Gestión empresarial desde la perspectiva epistemológica}

La ciencia de la administración ha evolucionado a través del tiempo por diversos criterios u obstáculo epistemológico en la construcción de nuevos conceptos, teorías y conocimientos (Contreras, 2010) de la siguiente manera: La ausencia y carencia de reflexión teórica en el campo. Aplicación de técnicas de investigación bajo criterios de usos y costumbres de la ciencia, sin cuestionarse sobre la pertinencia en los objetos de estudios. Escalonadas jerarquías de Autoridad-Responsabilidad de la estructura de poder académico, que muchas veces desconocen la realidad empresarial e implicaciones sociales. Ausencia de crítica y autocrítica como vigilancia epistemológica de la ciencia administrativa. Residuos aportes del Management y subordinación del campo académico al campo de los negocios.

\section{Teorías administrativas y gestión empresarial}

Hace más de dos décadas estudiosos e investigadores del pensamiento administrativo provenientes de diferentes escuelas académicos determinaron algunos /alcances sobre el conocimiento de la administración, en TCA, burocracia, relaciones humanas, comportamiento, sistemas sociales y otros. Estas escuelas conllevaron a un árbol de pensamiento administrativo confuso. En efecto, Koontz (2000) determino que estas 
confusiones estuvieron ligados a los diversos significados de palabras comunes como organización, la aparición de diferentes definiciones de la administración como un cuerpo de conocimientos, desechar los hallazgos de los aportes de la administración por considerar que fueron estudiados por practicantes pioneros desarrollados desde el café en lugar de valorar el espacio donde realmente fueron resaltado la pericia y pensamientos de hombres y mujeres ligados a la percepción administrativa, la incomprensión de la naturaleza y el rol de los principios de la administración así como la falta de voluntad de muchos expertos para entenderse mutuamente. Taylor, consideraba que lo importante es la producción y la eficiencia. ¿Qué hay del conocimiento en gestión empresarial? ¿Es más importante ser productivo que tener conocimiento en gestión empresarial?. La falta de gestión del conocimiento e innovación indican que una organización corra el riesgo de perder todo en un gran cambio en el ambiente productivo. El conocimiento dinamiza la gestión empresarial y la existencia de sus recursos es contrario a la producción de Taylor. El siglo XXI, no es igual al sector industrial del siglo XX, ahora se prioriza y da valor al conocimiento, debido a que somos una sociedad más exigente, reflexiva que cambia sus gustos y preferencias constantemente.

\section{Elementos de gestión empresarial}

\section{Gestión del conocimiento}

De acuerdo a (Pérez \& Coutín, 2005) la gestión del conocimiento es " una factor que planifica, organiza, dirige y controla los flujos del conocimiento que se producen en la empresa en relación con sus actividades productivas y con su entorno social con el propósito n de crear ciertas competencias esenciales". El conocimiento es inherente a los seres humanos y reside solo en la mente de las personas. La relación humano conocimiento es muy fuerte debido que el capitalismo intelectual relaciona las tecnologías y el conocimiento con el rol del capital humano que genera valor agregado (Hernández, 2011). Cuando una organización desea proporcionar servicios o productos, que satisfagan una necesidad con un alto valor agregado, para mantener ante sus usuarios/clientes una ventaja competitiva estable, debe saber utilizar y aprovechar los activos o intangibles que posee, específicamente, su cartera de conocimientos, porque estos constituyen, entre otros, la principal fuente de valor de la organización que garantice una buena gestión empresarial. En la actualidad, con la introducción de las nuevas tecnologías de información y comunicación, las organizaciones dependen cada vez más del uso que sean capaces de hacer de la información/conocimiento 
y de la capacidad de respuesta que tengan a las demandas cada vez más específicas y exigentes del mercado.

\section{Gestión de la información (GI)}

Cuando se refiere de organización económica o social productora de bienes y servicios, es de interés hablar de información. Un conocimiento que no se emplea no se convierte en información con ventaja competitiva, una información que se transmite se convierte en conocimiento con valor agregado. Rojas (2014), refiere que la gestión de información es el procedimiento que permite utilizar los recursos necesarios como: Gestión del talento humano, económicos y materiales para administrar la información desde sus inicios hasta la disposición final. Tiene como elemento básico, la gestión del ciclo de vida de este recurso y se desarrolla en cualquier organización. De esta manera el proceso de obtener la información parte desde su extracción, selección y distribución a los interesados.

Por ello, se desarrolla en unidades especializadas que administran estos recursos en forma intensiva, llamadas unidades de información. La GI en particular, se desarrolla en unidades especializadas que manejan este recurso en forma intensiva, llamadas unidades de información. Utiliza a las personas, la tecnología de la información y los procesos administrativos como herramienta de gestión empresarial para mejorar la productividad, la capacidad de liderazgo y las oportunidades de negocio con el fin de dinamizar la ventaja competitiva de la empresa (Naranjo, González, \& Rodríguez, 2016).

\section{Gestión por valores}

La cultura organizacional representa un patrón complejo de creencias, expectativas, ideas, valores, actitudes y conductas compartidas por los integrantes de una organización. Para (Quemada, 2016), los valores son aquellos en lo que creemos y con arreglo a lo cual nos comportamos. No se crean en una reunión de estrategia, pues ya están en la organización; la clave es descubrirlos, expresarlos y vivirlos. Los valores refuerzan la misión de la empresa, debiendo ser coherentes y estar alineados con esta. Los clientes se identifican con los valores de la empresa, porque piensan que son los suyos. Apple y Wal-Mart son claros ejemplo de esta práctica de valor. Estas compañías exitosas en el largo plazo viven marcadas por unos valores que no varían y un propósito que no cambian. El fundador de Walt Disney, por ejemplo, estableció como valores la imaginación y transmitir el bien a través de sus 
películas. Esto ha sido una guía central que ha marcado un rumbo para Disney en todos sus años de existencia.

El gran desafío de la gestión por valores es, entonces, la motivación inteligente, entendida como aquella que es capaz de valorar al sujeto y motivarlo a partir de sus propios intereses. Parte del sujeto, no del objeto, y por eso exige un mayor esfuerzo por parte de la organización, puesto que implica un conocimiento profundo de sus miembros a efectos de poder mostrar que esos valores y creencias que ellos tienen se alinean con los fines de la empresa. Por esta razón, el éxito en la aplicación de la dirección por valores estará dado en gran medida por lograr encontrar aquellos valores que aporten a las personas un sentido con el cual coincidan (Toniut et al., 2016).

\section{Dinamización empresarial}

El dinamismo empresarial se caracteriza por ser una fuerza activa que promueve una acción de cambio e innovación en la actividad económica que realiza la organización. (Vilariño, 2013) en las ciencias económicas la dinamización se da en el área de la comunicación y en la gestión empresarial como mecanismo integrador que demuestra el horizonte de transversalidad de cada sistema de gestión, siendo las personas o equipos de trabajo quienes generan mejoras, cambios e innovaciones en el negocio. Si se contempla la dinamización de la gestión empresarial de manera integral la administración de una organización toma decisiones sobre la base económica y financiera.

De acuerdo a Alizo y Escalona (2012), gestión económica (GE), emplea los recursos escasos para producir productos con mayor competitividad. Su competitividad está en el uso eficiente de los elementos del proceso de la administración y de sus subsistemas que activan el emprendimiento de la actividad económica de las organizaciones y diferencia su participación en el mercado globalizado. Este tipo de gestión es un siamés de la gestión financiera (GF) que según Cabrera, Fuentes y Cerezo, (2017) es un proceso de gestión y gerencia de cada organización al margen de su tamaño y sector al que pertenezca la empresa. El propósito de la gestión financiera es hacer que lo planificado se cumpla con eficiencia y eficacia, ya que se trata de propiciar un manejo técnico y transparente en el proceso de gestión empresarial y la asignación de los recursos financieros en las organizaciones.

En este escenario GE y GF tienen como socio de gestión a la toma de decisiones porque, a través de ella, la administración del negocio decide, por ejemplo, la puesta en marcha de las mejoras productivas, comerciales o distributivas. Según (Acuña et al., 2019) la toma de decisiones se halla en cada actividad que el hombre realiza dentro de las 
organizaciones sociales y económicas y se emplea como un instrumento organizacional que se toma a lo largo de la vida útil de la empresa.

\section{Metodología}

Por las particularidades de la investigación, el estudio fue cuantitativo y diseño descriptivo no experimental. La muestra fue no probabilística por conveniencia donde todos los encuestados de la población tuvieron iguales oportunidades de ser seleccionados. La recolección de datos fue directamente a los propietarios y familias que tuvieron capacidad de decisión en las MYPEs de confecciones del Parque Industrial de Ate-Vitarte. Para la obtención de la información se utilizó el cuestionario. La primera variable Gestión Empresarial conto con 15 preguntas las cuales 10 preguntas correspondieron a la dimensión Gestión del Conocimiento e Información y 5 preguntas a la dimensión Gestión del Valor. Su alfa de Cronbach fue de 0.881. Para la segunda variable Dinamización del desarrollo de las MYPEs se empleó 11 preguntas de las cuales 3 preguntas correspondieron a la dimensión Gestión Económica, 5 a la dimensión Gestión Financiera y 3 a la dimensión Toma de Decisiones. Su alfa de Cronbach fue de 0.827. Para el procesamiento y análisis comparativo de los datos fueron a través de los programas informáticos de Excel y el paquete estadístico SPSS versión 22 con la aplicación del análisis de regresión lineal múltiple; y el análisis descriptivo de las variables utilizando criterios estadísticos para variables cuantitativos.

\section{Resultados}

\section{Relación de gestión empresarial y sus dimensiones integradores en el pensamiento administrativo}

En la tabla 2, se observa el análisis de correlación Rho de Spearman para distribuciones no normales. La relación entre la Gestión empresarial (GE)y la Gestión de Conocimiento e Información (Gci) fue de $r=0.952(\mathrm{p}<0.01)$. La relación entre GE y la Gestión de Valor $(\mathrm{Gv})$ fue de $\mathrm{r}=0.856$ ( $\mathrm{p}$ < 0.01). La relación entre GE y Gestión E (Ge) fue de $r=0.832(p<0.01)$. La relación entre GE y Gf fue de $r=0.835(p<0.01)$ y la relación entre GE y la Toma de Decisiones fue de $r=0.717(\mathrm{p}<0.01)$.

La relación entre la GE y sus dimensiones es alta y significativa. La razón que explica este hecho se basa fundamentalmente que el proceso administrativo es aplicado en un entorno productivo ligado más a una experiencia empresarial practicado por 
iniciativa propia y heredado de sus padres y familiares que conocimientos académicos. La gestión empresarial cuyos elementos administrativos vincula a la planeación, organización, dirección y control dados por Fayol (1841-1925), a pesar de ser empleados empíricamente lo usan como un instrumento empresarial para dinamizar su actividad económica. Resaltan la obtención de conocimientos e información como parte de su giro de negocio, valoran la práctica de los principios axiológicos como el valor para ganar la confianza de sus clientes y posicionamiento a largo plazo de sus productos en el mercado. Además, su inminente juventud de los propietarios de las MYPEs de confecciones del Parque Industrial de Ate, Vitarte, les permiten aplicar la teoría Y de Douglas Mc Gregor porque se caracterizan por ser ser innovadores y asumir riesgos de inversión y financiamiento para producir las prendas de vestir en términos de calidad y a precios competitivos.

Tabla 2

Correlaciones de Gestión Empresarial y sus dimensiones

\begin{tabular}{lcccccc}
\hline \multicolumn{1}{c}{ Gestión Empresarial } & 1 & 2 & 3 & 4 & 5 & 6 \\
\hline Gestión de conocimiento & 1.000 & & & & & \\
e información [1] &, $756^{* *}$ & 1.000 & & & & \\
Gestión de valor [2] &, $735^{* *}$ &, $694^{* *}$ & 1.000 & & & \\
Gestión económica [3] &, $725^{* *}$ &, $695^{* *}$ &, $669^{* *}$ & 1.000 & & \\
Gestión financiera [4] &, $574^{* *}$ &, $654^{* *}$ &, $631^{* *}$ &, $609^{* *}$ & 1.000 & \\
Toma de decisiones [5] &, $952^{* *}$ &, $856^{* *}$ &, $832^{* *}$ &, $835^{* *}$ &, $717^{* *}$ & 1.000 \\
\hline
\end{tabular}

**. La correlación es significativa en el nivel 0,01 (bilateral).

\section{Gestión de conocimiento e información en gestión empresarial y su aplicación del pensamiento administrativo}

En la tabla 3, se evidencia que la dimensión de Gci se aplica en GE por medio de los modelos del pensamiento científico de la administración, de un modo empírico: Un 19\% (10p) consideran que la planificación de Taylor (1856-1915) es importante para orientar a la empresa alcanzar sus objetivos, adquirir nuevos conocimientos para diseñar nuevos productos y racionalizar la producción con eficiencia en la gestión empresarial. Un 43\% (22p) manifestaron que planifican y organizan las actividades en base a sus conocimientos administrativos, técnicos, comerciales, financieras y contables de acuerdo a la teoría de Fayol (1841-1925). Un 19\% (10p) cuentan con un presupuesto para invertir en publicidad 
de sus productos. Un 45\% (23p) coordinan sus actividades administrativas y productivas para alcanzar sus objetivos. Un 56\% (28p) delega autoridad a sus familiares con quienes comparte la administración de la empresa con el fin de compartir las tareas y dedicarse en asuntos que requiere mayor atención.

Claro empleo de la teoría de las relaciones humanas de Munsterberg y Elton Mayo (1924-1932) e integración social. Un 43\% (22p) verifica si toda la actividad ocurre de conformidad con el plan adoptado y de acuerdo al criterio de Fayol. Un 30\% (15p) racionalizan el trabajo de sus obreros para mejorar la productividad poniendo en manifiesto la aplicación de la teoría de Taylor. Un 26\% (13p) dividen el trabajo con la finalidad de especializar el trabajo por áreas específicas de acuerdo a Taylor. Un 20\% (10p) interactúan en la comunidad satisfaciendo las necesidades de la sociedad y de los clientes internos relevando la participación de la teoría de Maslow y Douglas Mc Gregor (1970). Un 48\% (24p) satisfacen las necesidades de los trabajadores de acuerdo a la naturaleza y condiciones del trabajo.

La evaluación de estos resultados está dada sobre la base máxima de 50 puntos de la dimensión de Gci encuestadas. Lo que significa que las teorías del pensamiento administrativo que guardan relación con GCI se aplican en la GE de las MYPEs de confecciones del Parque Industrial de Ate, Vitarte. A pesar de contar con un nivel de instrucción primaria y secundaria entienden por experiencia propia y heredada de los padres $\mathrm{y}$ familiares que Gci es un proceso que les ha permitido ir acumulando conocimientos e información a través del tiempo y generar fuerte identidad con sus organizaciones y clientes. Además, se han adaptado a las exigencias de competitividad del mercado asegurando sus inversiones con retornos a corto plazo y orientar sus negocios con nuevas perspectivas empresariales. 


\section{Tabla 3}

Evaluación porcentual de gestión de conocimiento e información

\begin{tabular}{|c|c|c|c|c|c|c|c|}
\hline Ítems & $\begin{array}{c}\text { Muy en } \\
\text { desacuerdo }\end{array}$ & $\begin{array}{c}\text { En } \\
\text { desacuerdo }\end{array}$ & $\begin{array}{c}\mathrm{Ni} \text { de } \\
\text { acuerdo, ni } \\
\text { en } \\
\text { desacuerdo }\end{array}$ & De acuerdo & $\begin{array}{l}\text { Muy de } \\
\text { acuerdo }\end{array}$ & Promedio & $\begin{array}{l}\text { Desviación } \\
\text { Estándar }\end{array}$ \\
\hline \multicolumn{8}{|c|}{ Gestión de conocimiento e información $($ Promedio $=3.7)$} \\
\hline gci_1 & $5.9 \%$ & $18.8 \%$ & $16.8 \%$ & $18.8 \%$ & $39.6 \%$ & 3.7 & 1.3 \\
\hline gci_2 & $5.9 \%$ & $20.8 \%$ & $17.8 \%$ & $42.6 \%$ & $12.9 \%$ & 3.4 & 1.1 \\
\hline gci_3 & $8.9 \%$ & $31.7 \%$ & $10.9 \%$ & $18.8 \%$ & $29.7 \%$ & 3.3 & 1.4 \\
\hline gci_5 & $0.0 \%$ & $0.0 \%$ & $6.9 \%$ & $44.6 \%$ & $48.5 \%$ & 4.4 & 0.6 \\
\hline gci_6 & $0.0 \%$ & $0.0 \%$ & $7.9 \%$ & $56.4 \%$ & $35.6 \%$ & 4.3 & 0.6 \\
\hline gci_7 & $0.0 \%$ & $0.0 \%$ & $5.9 \%$ & $42.6 \%$ & $51.5 \%$ & 4.5 & 0.6 \\
\hline gci_8 & $5.0 \%$ & $20.8 \%$ & $33.7 \%$ & $29.7 \%$ & $10.9 \%$ & 3.2 & 1.1 \\
\hline gci_9 & $5.9 \%$ & $19.8 \%$ & $38.6 \%$ & $25.7 \%$ & $9.9 \%$ & 3.1 & 1.0 \\
\hline gci_11 & $7.9 \%$ & $36.6 \%$ & $25.7 \%$ & $19.8 \%$ & $9.9 \%$ & 2.9 & 1.1 \\
\hline gci_13 & $0.0 \%$ & $1.0 \%$ & $23.8 \%$ & $48.5 \%$ & $26.7 \%$ & 4.0 & 0.7 \\
\hline
\end{tabular}

\section{Gestión de valor en gestión empresarial y su aplicación del pensamiento administrativo}

En la tabla 4, muestra que un 34\% (9p) de los propietarios de las MYPEs del área de confecciones consideran que las actividades del negocio evidencian el compromiso que tienen los trabajadores en cumplir con la misión y búsqueda de metas de la empresa considerando la teoría de Douglas Mc Gregor. Un 17\% (4p) están de acuerdo que al momento de contratar un trabajador asume el propietario la responsabilidad de desarrollo profesional de su personal. Un 75\% (19p) opinaron que la trasparencia es relevante por su énfasis en las personas y el cambio social que fortalece la vida organizacional; y por el posicionamiento de sus negocios en el mercado que postula la teoría de del Desarrollo Organizacional (DO) de Bennis, Blaki y Shepard (1958). Un 69\% (17p) afirmaron que la confianza es un indicador que garantiza las operaciones comerciales con los clientes. Un 45\% (11p) manifestaron que el mercado competitivo exige la identificación de los trabajadores con la empresa para generar ventaja competitiva.

Si se considera estos resultados sobre la base de un máximo de 25 puntos de la dimensión Gv encuestadas se aplican en la GE por medio del proceso administrativo 
constituyendo para los propietarios de las MYPEs en un insumo valioso, porque sumara a lo que ya están practicando establecer nuevas estrategias y políticas que garantizaran transacciones comerciales con sus clientes y proveedores honestas y confiables. Además, la tenencia del valor será visto como una convicción, una creencia abordado como una intangibilidad servirá para que sus empresas orienten sus acciones productivas con mayor capacidad en GE y ventaja comparativa ante sus competencias.

\section{Tabla 4}

Evaluación porcentual de gestión de valor

\begin{tabular}{|c|c|c|c|c|c|c|c|}
\hline Ítems & $\begin{array}{c}\text { Muy en } \\
\text { desacuerdo }\end{array}$ & $\begin{array}{c}\text { En } \\
\text { desacuerdo] }\end{array}$ & $\begin{array}{c}\text { Ni de } \\
\text { acuerdo, ni } \\
\text { en } \\
\text { desacuerdo }\end{array}$ & De acuerdo & $\begin{array}{l}\text { Muy de } \\
\text { acuerdo }\end{array}$ & Promedio & $\begin{array}{c}\text { Desviación } \\
\text { Estándar }\end{array}$ \\
\hline
\end{tabular}

Gestión de valor (Promedio = 3.9)

$\begin{array}{lccccccc}\text { gv_1 } & 0.0 \% & 1.0 \% & 9.9 \% & 33.7 \% & 55.4 \% & 4.4 & 0.7 \\ \text { gv_3 } & 16.8 \% & 33.7 \% & 5.0 \% & 16.8 \% & 27.7 \% & 3.0 & 1.5 \\ \text { gv_6 } & 0.0 \% & 1.0 \% & 5.0 \% & 75.2 \% & 18.8 \% & 4.1 & 0.5 \\ \text { gv_7 } & 0.0 \% & 0.0 \% & 4.0 \% & 69.3 \% & 26.7 \% & 4.2 & 0.5 \\ \text { gv_8 } & 0.0 \% & 3.0 \% & 33.7 \% & 45.5 \% & 17.8 \% & 3.8 & 0.8\end{array}$

\section{Gestión económica en gestión empresarial y su aplicación del pensamiento administrativo}

En la tabla 5, se evidencia que un 37\% (6p) de los propietarios de las MYPEs del área de confecciones, consideran que el capital de trabajo lo invierten para priorizar la tenencia de existencias considerando los criterios del proceso administrativo. La inversión para estas empresas es una Entropía (teoría de los sistemas de Von Bertalanffy (1968) por carecer de orientación profesional en priorizar la inversión en capital de operaciones o capital fijo y por qué sus sistemas dependen más de sus criterios empresariales que de su estructura organizacional. Un $28 \%$ (4p) manifestaron que los costos de producción son trabajados con mano de obra calificada para lograr eficiencia en la productividad según la teoría de Taylor. Un 68\% (10p) de los encuestados manifestaron que la tenencia de los inventarios facilita producir las prendas de vestir según los requerimientos de sus segmentos de mercado. El 
control como parte de los elementos de administración dado por Fayol se evidencia en el proceso de producción de las prendas de vestir que producen las MYPEs.

Si se considera estos resultados sobre la base de un máximo de 15 puntos de la dimensión Ge encuestadas significa que el proceso administrativo es aplicado en la gestión empresarial. Lo que influye en a las MYPEs dinamizar el aparato productivo de sus negocios, acumular ingresos y ser forjadores de fuentes de empleo directos e indirectos a pesar de sus limitados conocimientos de las teorías administrativas.

Tabla 5

Evaluación porcentual de gestión económica

\begin{tabular}{lccccccc}
\hline Ítems & $\begin{array}{c}\text { Muy en } \\
\text { desacuerdo }\end{array}$ & $\begin{array}{c}\text { En } \\
\text { desacuerdo }\end{array}$ & $\begin{array}{c}\text { Ni de } \\
\text { acuerdo, ni en } \\
\text { desacuerdo }\end{array}$ & De acuerdo & $\begin{array}{c}\text { Muy de } \\
\text { acuerdo }\end{array}$ & Promedio & $\begin{array}{c}\text { Desviación } \\
\text { Estándar }\end{array}$ \\
\hline Gestión económica (Promedio $=4.1)$ & & & & & \\
ge_1 & $0.0 \%$ & $0.0 \%$ & $4.0 \%$ & $36.6 \%$ & $59.4 \%$ & 4.6 & 0.6 \\
ge_3 & $4.0 \%$ & $23.8 \%$ & $9.9 \%$ & $27.7 \%$ & $34.7 \%$ & 3.7 & 1.3 \\
ge_4 & $0.0 \%$ & $2.0 \%$ & $12.9 \%$ & $68.3 \%$ & $16.8 \%$ & 4.0 & 0.6 \\
\hline
\end{tabular}

\section{Gestión financiera en gestión empresarial y su aplicación del pensamiento administrativo}

En la tabla 6, se evidencia que un 20\% (5p) de las personas encuestadas manifestaron que los registros contables viabilizan las inversiones para la sostenibilidad de la empresa. La teoría clásica de la administración dada por Fayol resalta en este resultado, particularmente el uso de los elementos de la administración. Un 52\% (13p) indicaron que el uso de los activos corrientes a corto plazo les permite maximizar el soporte financiero del negocio. El pensamiento administrativo de Fayol se evidencia en este resultado por su característica de cumplir con la función financiera, seguridad y contable. Un 49\% (12p) manifestó que la disponibilidad financiera cubre los pasivos corrientes de la empresa a pesar de contar con escasa diversificación en su estructura organizacional de tipo familiar no burocrática la que postula Max Weber (1864-1920) son eficientes y eficaces en su paradigma de participar en el mercado.

Un $51 \%$ (13p) estuvieron de acuerdo que el ciclo productivo del negocio garantiza la rentabilidad del negocio a pesar de contar con escasa diversificación organizacional. ¿Es 
una entropía? Las MYPEs tienen una perspectiva más alta de mantener estructuras organizacionales más eficientes y técnicas como la que postula la teoría estructuralista de Burnham (1950). Un 50\% (13p) confirmo que la liquidez económica garantiza apalancar la obtención de nuevos recursos financieros debido al empleo de los elementos de la administración dados por Taylor y Fayol.

Al considerar estos resultados y tomando en cuenta un máximo de 25 puntos de la dimensión Gf encuestadas se concluye que el proceso administrativo se aplica en la Gf y con influencia en la GE que desarrollan los propietarios de las MYPEs de confecciones. Estos empresarios a pesar de contar con un limitado capital y limitado conocimiento en las ciencias económicas utilizan la gestión financiera y sus fuentes de financiamiento de manera prudente y eficaz en sus respectivos gastos y costos de producción de sus productos.

\section{Tabla 6}

Evaluación porcentual de gestión financiera

\begin{tabular}{|c|c|c|c|c|c|c|c|}
\hline Ítems & $\begin{array}{c}\text { Muy en } \\
\text { desacuerdo }\end{array}$ & $\begin{array}{c}\text { En } \\
\text { desacuerdo }\end{array}$ & $\begin{array}{c}\text { Ni de } \\
\text { acuerdo, ni } \\
\text { en } \\
\text { desacuerdo }\end{array}$ & De acuerdo & $\begin{array}{l}\text { Muy de } \\
\text { acuerdo }\end{array}$ & Promedio & $\begin{array}{c}\text { Desviación } \\
\text { Estándar }\end{array}$ \\
\hline
\end{tabular}

Gestión financiera (Promedio $=4.0)$

\begin{tabular}{lccccccr} 
gf_1 & $5.9 \%$ & $20.8 \%$ & $16.8 \%$ & $19.8 \%$ & $36.6 \%$ & 3.6 & 1.3 \\
gf_2 & $0.0 \%$ & $7.9 \%$ & $19.8 \%$ & $52.5 \%$ & $19.8 \%$ & 3.8 & 0.8 \\
gf_3 & $0.0 \%$ & $0.0 \%$ & $5.9 \%$ & $49.5 \%$ & $44.6 \%$ & 4.4 & 0.6 \\
gf_5 & $0.0 \%$ & $0.0 \%$ & $3.0 \%$ & $51.5 \%$ & $45.5 \%$ & 4.4 & 0.6 \\
gf_6 & $0.0 \%$ & $5.9 \%$ & $26.7 \%$ & $50.5 \%$ & $16.8 \%$ & 3.8 & 0.8 \\
\hline
\end{tabular}

\section{Toma de decisiones en gestión empresarial y su aplicación del pensamiento administrativo}

En la tabla 7, se evidencia que un 34\% (5p) de los propietarios de las MYPEs encuestados manifestaron que están de acuerdo que la toma de decisiones oportuna les permite alcanzar rentabilidad en sus respectivas empresas. Su base empírica está centrada en los criterios del pensamiento administrativo de Taylor, Fayol, Elton Mayo, Douglas Mc Gregor y de Bennis, Blake y Shepard. Un 45\% (7p) manifestaron que la presentación de oportunidades de negocio toma decisiones considerando los criterios del proceso administrativo de Fayol. Un 48\% (7p) consideraron que cuando identifican un problema en 
el desarrollo de sus actividades económicas selecciona alternativas de solución como parte de su gestión empresarial.

Con los resultados hallados y tomando en cuenta un máximo de 15 puntos de la dimensión Td encuestadas se concluye que el proceso administrativo se aplica en la $\mathrm{Td}$ y con influencia en la GE que desarrollan los empresarios confeccionistas. Para ellos la Td es considerado como una alternativa adecuada porque les permiten decidir día a día como deben hacer frente sus problemas administrativos, económicos, financieros, comerciales, logísticos, etc que en la mayoría de los casos imposibilitan su desarrollo dada sus limitaciones de recursos. Asimismo, son conscientes que sus soluciones no son de empresario económico, sino de Micro y Pequeños empresarios administrativos que buscan soluciones satisfactorias que influyan en sus objetivos organizacionales.

\section{Tabla 7}

Evaluación porcentual de toma de decisiones

\begin{tabular}{|c|c|c|c|c|c|c|c|}
\hline Ítems & $\begin{array}{c}\text { Muy en } \\
\text { desacuerdo }\end{array}$ & $\begin{array}{c}\text { En } \\
\text { desacuerdo }\end{array}$ & $\begin{array}{c}\text { Ni de } \\
\text { acuerdo, ni } \\
\text { en } \\
\text { desacuerdo }\end{array}$ & De acuerdo & $\begin{array}{l}\text { Muy de } \\
\text { acuerdo }\end{array}$ & Promedio & $\begin{array}{l}\text { Desviación } \\
\text { Estándar }\end{array}$ \\
\hline \multicolumn{8}{|c|}{ Toma de decisiones $($ Promedio $=4.2$ ) } \\
\hline td_1 & $0.0 \%$ & $0.0 \%$ & $3.0 \%$ & $33.7 \%$ & $63.4 \%$ & 4.6 & 0.5 \\
\hline td_2 & $0.0 \%$ & $1.0 \%$ & $27.7 \%$ & $45.5 \%$ & $25.7 \%$ & 4.0 & 0.8 \\
\hline $\operatorname{td} \_4$ & $0.0 \%$ & $1.0 \%$ & $19.8 \%$ & $48.5 \%$ & $30.7 \%$ & 4.1 & 0.7 \\
\hline
\end{tabular}

\section{Modelo de gestión empresarial}

El modelo de Gestión Empresarial fue revisado a través de un Análisis Factorial Exploratorio (AFE), en el cual se comprobaron las 5 dimensiones, y sus respectivos indicadores. Sin embargo, al no tener todos los indicadores una correlación lineal alta y significativa, se trabajó con el software Amos v24 y el Análisis Factorial Confirmatorio (AFC), para evidenciar la validez del constructo y mejorar el modelo de GE. Se empleó las escalas tipo Likert con base en los índices $\mathrm{x}^{2}$ de razón de verosimilitud y RMSEA de $0.05<$ 0.8 que es un ajuste razonable. El número de factores o dimensiones inicialmente fueron con 39 preguntas (13p Gci, 8p Gv, 6p Ge, 6p Gf y 6p Td) al no encontrarse preguntas que no se correlacionaban entre sí se empleó el AFC con el fin de reducir las preguntas a 26 (10p Gci, 
5p Gv, 3p Ge, 5p Gf y 3p Td) y se encontró grupos homogéneos con significado común que se correlacionaban evitando de esta manera trabajar con grupos independientes.

La figura 4, evidencia el modelo ajustado de GE y su relación con la Gci. Esta dimensión resalta su importancia porque los propietarios de las MYPEs de Confecciones, emplean las teorías del pensamiento de la administración clásica de Fayol, haciendo uso de los elementos del proceso administrativo en la GE y la adquisición de conocimientos e información para diseñar en mejores condiciones sus productos y diferenciar la productividad con sus competencias. Además, emplean los criterios del razonamiento de la administración científica de Taylor y de su teoría de la productividad y eficiencia en el trabajo.

También emplean el pensamiento administrativo de otros personajes de la administración como: Max Weber cuya teoría se orienta en la estructura funcional cerrada e importancia de la planeación y el control. Hugo Munterberg y Elton Mayo y su aporte sobre las relaciones interpersonales e integración social. Abram Maslow y Douglas Mc Gregor y la satisfacción de las necesidades. Benner, Blake y Shepard y sus énfasis en la relación entre las personas y la organización y la práctica de la cultura organizacional en los negocios. Todas estas teorías también son empleados por los propietarios de MYPEs en la GE por experiencia empresarial que por conocimientos académicos.

En segundo lugar, la GE se relaciona con Gv en la medida que sean transparente e inspiren confianza en sus operaciones mercantiles que llevan a cabo con sus clientes y proveedores garantizaran la venta de sus producciones y posicionarse en el mercado a largo plazo. A la vez, cumplen con la misión de sus organizaciones y asumen relativamente con la responsabilidad de desarrollo profesional de sus trabajadores.

En tercer lugar, la GE se relaciona con Gef resaltando el empleo del capital de trabajo e inversión en la tenencia de las existencias y la disposición de los flujos de efectivo que garantizan a los propietarios de las MYPEs disponer del soporte financiero y cubrir sus pasivos corrientes a corto plazo, hacer uso del ciclo productivo, obtener utilidad y apalancar nuevos recursos para dinamizar con mayor contundencia sus actividades económicas en el largo plazo. En cuarto lugar, la GE se relaciona con la Td porque consideran los propietarios de las MYPEs que es un instrumento que les abre espacios de aprendizaje y acumulación de informaciones para tomar decisiones adecuadas del que hacer empresarial y de permanencia en el mercado. 


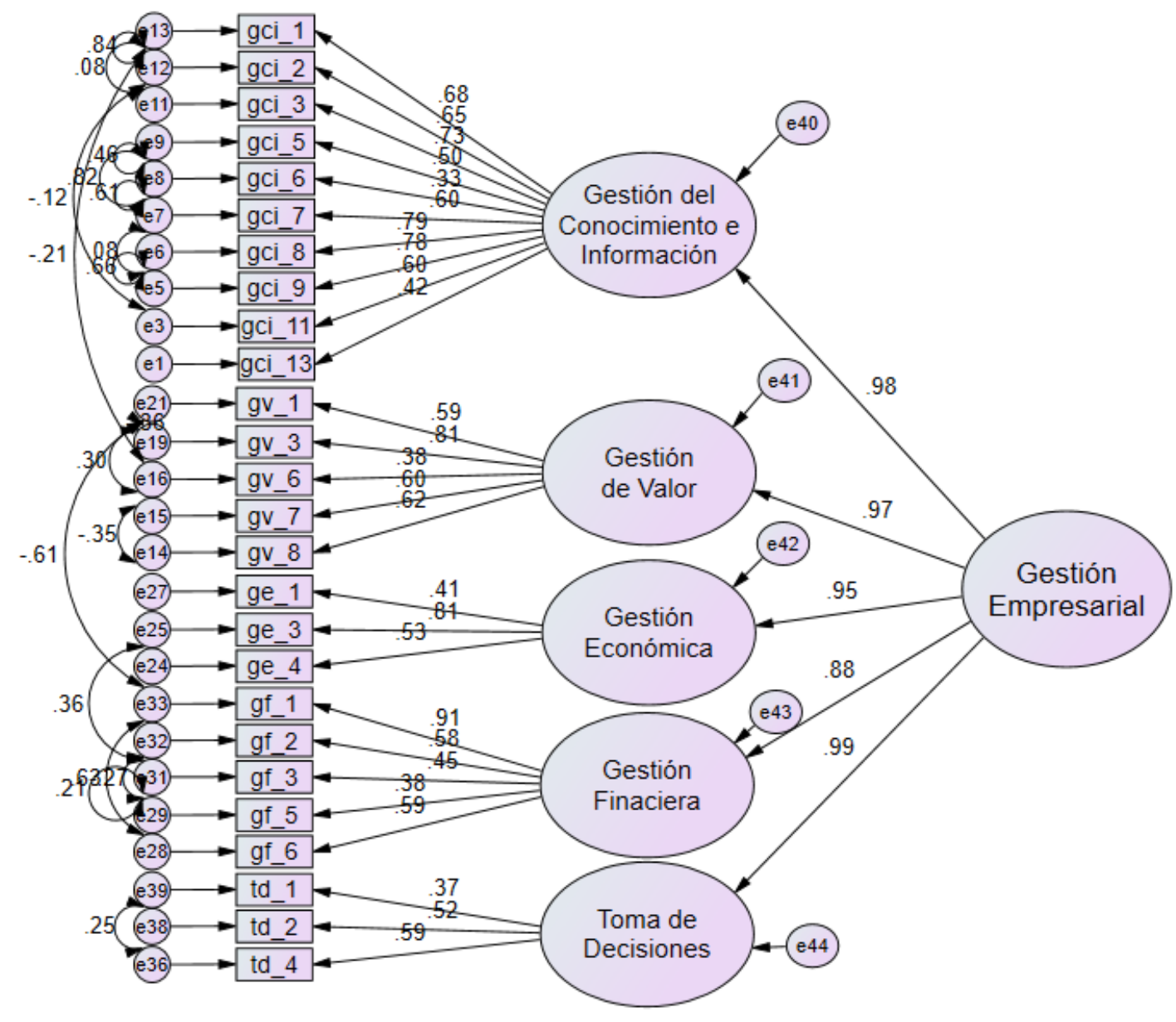

Figura 4. Modelo ajustado de Gestión Empresarial

Tomando en cuenta las ecuaciones 1 - 5 y la figura 4 , también este modelo se puede expresar matemáticamente empleando la teoría del pensamiento administrativo de Henry Fayol y de su análisis del proceso administrativo (planificación, organización, dirección y control) toda vez que estos elementos está integrada en todas las dimensiones de GE de la siguiente manera:

Considerando la función de varias variables del proceso administrativo (PA) representado por: $f: I R^{4} \longrightarrow I R$

$(\mathrm{Pa}, \mathrm{Oo}, \mathrm{Di} . \mathrm{Co}, \xi) \longrightarrow \quad \mathrm{Y}=(\mathrm{Pa}, \boldsymbol{O o}, \mathrm{Di}, \mathrm{Co}, \xi)$ cuya regla de correspondencia es definida por la siguiente ecuación:

$f(\mathrm{~Pa}, \mathrm{Oo}, \mathrm{Di}, \mathrm{Co}, \xi)=\beta_{0}+\beta_{1} \mathrm{~Pa}_{+} \beta_{2} \mathrm{O}_{O}+\beta_{3} \mathrm{Di}+\beta_{4} \mathrm{Co}+\beta_{1} \xi+U$

En este modelo, la planificación $(\mathrm{Pa})$, la organización $(\mathrm{Oo})$, la dirección $(\mathrm{Di})$ y el control $(\mathrm{Co})$ es medido por el parámetro de Gestión Empresarial $(G E)$ y por el parámetro de 
la variabilidad de otros factores $(\xi)$ como la integración y demás elementos del pensamiento administrativo. Las variaciones de estas variables son explicadas por el cambio que pueden realizar los propietarios de las PYMEs de confecciones del parque industrial de Ate. Vitarte, a través de la práctica de una eficiente GE aun cuando podría existir otras variables o perturbaciones aleatorias (u) que podrían actuar aisladamente en ella. Ahora bien, se estudia GE porque asume el rol de variable integrador que dinamiza el desarrollo de la actividad económica de las PYMEs cuya función está dada por:

\section{$G E: I R^{5} \longrightarrow I R$}

$(G c i, G v, G e, G f, T d) \longrightarrow Y=G E(G c i, G v, G e, G f, T d)$ cuya regla de correspondencia es una función multivariada de potencias homogéneas de grado $\mathbf{a}+\mathbf{b}+\mathbf{c}+\mathbf{d}+\mathbf{e}$ denotado por:

$G E(G c i, G v . G e, G f, T d)=A(G c i)^{a}(G v)^{b}(G e)^{c}(G f)^{d}(T d)^{e}$

$$
\text { Gci }>0, G v>0, G e>0, G f>0, T d>0 \quad A, a, b, c, d, e=\text { Constantes }
$$

En el modelo (7), la GE por su importancia de la viabilidad de la administración del negocio de las PYMEs depende de la capacidad emprendedora de sus propietarios para emplear con mayor ventaja competitiva la gestión del conocimiento e información (Gci), gestión del valor $(G v)$, gestión económica $(G e)$, gestión financiera $(G f)$, y toma de decisiones (Td) en la organización. La $G E$ al integrar la $G c i$ y $G v$ desarrolla actividades nuevas y estratégicas basadas en la honestidad y confianza. Garantiza que la oferta satisfaga la demanda de las necesidades del consumidor con características competitivas ya que activa la $G f$, las inversiones y $T d$ a corto y largo plazo de las PYMes. Así la $G E$ se fundamenta en un modelo de regresión lineal múltiple definida en las en las ecuaciones (6) y (7), las cuales se orientan en una función multivariada definida por:

$G E: I R^{9} \longrightarrow I R$

(Pa, Oo, Di, Co,, , Gci, Gv, Ge, Gf, Td) $\quad Y=G E(P a, O o, D i, C o, \xi, G c i, G v, G e, G f$, Td)

cuya regla de correspondencia está compuesta por dos funciones definidas y positivas donde:

$Y=P A=F(P a, O o, D i, C o, \xi) G E(G c i, G v, G e, G f, T d)$

Donde:

$G E=\left[\beta_{0}+\beta_{1} P_{a}+\beta_{2} O o+\beta_{3} D_{i}+\beta_{4} C o+\beta_{1} \xi+U\right]\left[\mathrm{A}(\mathrm{Gci})^{\mathrm{a}}(\mathrm{Gv})^{\mathrm{b}}(\mathrm{Ge})^{\mathrm{c}}(\mathrm{Gf})^{\mathrm{d}}(\mathrm{Td})^{\mathrm{e}}\right]$ 
La ecuación (8) es el modelo de GE del objeto de estudio que hipotéticamente ayudará dinamizar el desarrollo social y económico de las MYPEs de confecciones del Parque Industrial de Ate, Vitarte.

\section{Conclusión}

Las diferentes concepciones de la evolución de las teorías del pensamiento administrativo trajeron como consecuencia la aparición de organización formal, cerrada y estructuralmente compleja. Se suma a este sistema el dar respuesta a los problemas de productividad y eficiencia laboral desde un punto de vista de la ingeniería y del supuesto de que la única motivación de la fuerza de trabajo en las empresas es la motivación económica (homo economicus), las buenas relaciones interpersonales, integración social y el desarrollo organizacional.

Los resultados muestran que los supuestos enfoques del pensamiento de la administración se aplican en la Gci, Gv. Ge, Gf y Td de las MYPEs de Confecciones del Parque Industrial de Ate, Vitarte, basado más por sus experiencias empresariales que conocimientos de las teorías administrativas. Influyen en la práctica de la GE que dinamiza la actividad económica de cada uno de sus organizaciones según sus características productivas. Además, mediante el análisis de regresión múltiple se pudo obtener un $\mathrm{r}^{2}$ ajustado significativo en cada una de las dimensiones de GE que va desde 0.495 a 0.898 . Finalmente, la toma de decisiones para estos Micros y Pequeños empresarios es relevante porque a través de ella les permiten decidir las nuevas inversiones y asumir el riesgo del costo-beneficio y seguir permaneciendo en el mercado

\section{Referencias}

Alizo, M., \& Escalona, M. (2012). Factores de gestión económica estratégica del emprendimiento tipo pyme, vinculados al mercado en venezuala. Cuadernos del Cendes, 29 (79), 1-34.

Acuña, C., Ortiz, J., Abad, M., \& Naranjo, E. (2019). Toma de decisiones en las pymes ecuatorianas: industrias de pichincha y azuay. Revista ESPACIOS, 40 (40), 18.

Beltán, L. (2020). Aportes del estructuralismo a la identificación del objeto de estudio de la comunicación 2008. Revista Razon y Palabra, 13 (63), 12.

Cabrera, C., Fuentes, M., \& Cerezo, G. (2017). La gestión financiera aplicada a las 
organizaciones. Dominio de las Ciencias, 3 (4), 220-232. https://doi.org/10.23857/dc.v3i4

Contreras, R. (2010). Reflexiones en torno a la construcción cintífica en el campo de los estudios de las organizaciones, gestión y administración en méxico. Revista Académica de Economía, (127), 1-25.

Chiavenato I. (1999). Introducción a la teoría general de la Administración. Mexico D. F.: Editorial McGraw Hill.

Espinoza, R. (2009). El fayolismo y la organización contemporánea. Visión Gerencial, (1), 53-62.

Gómez M. (2008). Diseño de estrategias que permitan a las organizaciones pasar a una posición de generación y difusión de conocimiento: Caso de las organizaciones del sector paraestatal [Tesis doctoral, Universidad Autónoma de Querétaro].

Hernández, H. (2011). La gestión empresarial, un enfoque del siglo XX, desde las teorías administrativas científica, funcional, burocrática y de relaciónes humanas. Escenarios, 9

$38-51$. https://dialnet.unirioja.es/metricas/documentos/ARTREV/3875234

Hernández S. y Rodríguez G. (2012). Administración: teoría, proceso, áreas funcionales y estrategias para la competitividad. México D. F.: Editorial Mc Graw Hill.

Hodson, W. (2001). Manual del ingeniero industrial (4ta ed.). México D. F.: Mc Graw Hill. Koonzt H. (2000). The management theory jungle academy of management journal Medina, A., \& Ávila, A. (2002). Evolución de la teoría administrativa. una visión desde la psicología organizacional. Revista Cubana de Psicología, 19 (3), 262-272.

Naranjo, S., González, D., \& Rodríguez, J. (2016). El reto de la gestión del conocimiento en las instituciones de educación superior colombianas. Administración Contemporánes. Revista de Investigación, 2 (44), 151-164. https://doi.org/https://doi.org/10.17227/01234870.44folios151.164

Niebel, B. y, \& Freivalds, A. (2009). Ingeniería industrial: Métodos, estándares y diseño del trabajo. México D. F.: Mc Graw Hill.

Pérez, Y., \& Coutín, A. (2005). La gestión del conocimiento : un nuevo enfoque en la gestión empresarial. Acimed, 13 (6), 74.

Ponce y salas (2014). Efecto del uso de la tecnología en las relaciones humanas en el ámbito institucional. Proyecto de investigación. Colombia: Universidad Nacional de Córdoba. 
Quemada, E. (2016). Estrategia, valor y precio: Maximizar el verdadero valor de la empresa. Madrid, España: Ediciones Pirámide.

Torres, Z. (2014). Teoría General de la Administración. México: Grupo Editorial Patria.

Toniut, H., del Valle, S., Dondero, M., Molina, D., Nivollet, D., Ordoñez, V., \& Sordo, J. (2016). La gestión por valores: una mirada crítica sobre la gestión de organizaciones. Revista Argentina de Investigación en Negocios, 1 (2), 2422-7609. https://ppct.caicyt.gov.ar/index.php/rain/article/download/v1n2a06/6659

Velásquez, F. (2002). Escuelas e interpretaciones del pensamiento administrativo. Estudios Gerenciales, (83), 31-55.

Vilariño, C. (2013). Gestión ambiental empresarial: contribución a su dinamización desde el diseño estratégico. Omnia, 19 (3), 11-19. 